\title{
手性金属有机框架材料(MOFs)的合成及应用
}

\author{
赵莉曾和平* \\ (华南理工大学化学与化工学院＼cjkstart发光材料与器件国家重点实验室 广州 510640)
}

\begin{abstract}
摘要 手性材料一直以来都是科学工作者研究的重点, 尤其是在药物和生物体中的应用方面. 手性金属有机框架材料 (MOFs) 具有迷人的拓补结构和潜在的应用价值, 受到了人们的广泛关注. 主要从它的合成和应用方面做了简要的介绍. 关键词 手性金属有机框架材料(MOFs); 模板; 不对称催化; 对映选择性分离
\end{abstract}

\section{Synthesis and Application of Chiral Metal-Organic Framework Materials (MOFs)}

\author{
Zhao, Li Zeng, Heping* \\ (State Key Laboratory of Luminescent Materials \& Devices, School of Chemistry \& Chemical Engineering, \\ South China University of Technology, Guangzhou 510640)
}

\begin{abstract}
Scientific workers have put the emphasis on the chiral material for a long time, especially in drugs and application of organisms. Chiral metal-organic framework materials (MOFs) have attracted significant attention because of not only their enormous variety of interesting structural topologies but also their wide potential applications. In this paper, its synthesis and application are introduced.
\end{abstract}

Keywords chiral metal-organic framework materials (MOFs); template; asymmetric catalysis; enantioselective separation

自 20 世纪 90 年代初期 Hoskins 和 Robson ${ }^{[1,2]}$ 开创 金属有机框架材料(MOFs)研究以来, 由于 MOFs 材料的 迷人的框架结构、高的孔隙率、好的化学稳定性、可再 生性、合成过程与仪器简单及其潜在的实用价值, 受到 广泛关注. 虽然由于结构表征以及性能测试方面的困 难, 增加了 MOFs 研究的难度, 但丝毫未降低其吸引 力 $^{[3 \sim 6]}$. 近十几年, MOFs 已经成为化学学科中发展最快 的领域之一.

金属有机框架化合物研究非常迅速，而手性 MOFs 由于手性配体合成难、价格贵, 非手性配体合成时结构 难预料等原因使其发展缓慢, 迄今为止有关手性 MOFs 的研究只相当于 MOFs 材料的 1\%左右. 手性催化 MOFs 最早取得的突破是通过 POST-1 获得的 ${ }^{[7]}$, 随后在其基 础上进行了改进, 得到了一系列不同的网状结构手性 MOFs 材料, 在对映体催化方面取得了很大的成功, 但 在均相催化方面还没有发现很好的晶体, 因此它仍然有 很大的发展潜力.
此外, 手性 MOFs 在很多领域变得越来越重要, 尤 其是与生命息息相关的行业像制药学、诊断分析、生物 工程等, 现已证明其在身体和环境中表现是不同的. 例 如: 有些药物有左、右旋两种, 其化学性质完全一样, 但 微观作用机理和药力、毒性等方面可能完全不同, 有些 药物中只有其中的一种分子结构对疾病有疗效，而其对 映异构体的疗效甚微或不起作用，甚至有剧毒. 正是由 于在生物医学中手性的重要性以及开发对映选择性的 手性沸石方面的工作进展缓慢 ${ }^{[8]}$ (虽然已经合成出能将 环氧丙醇的左右旋对映体分离的纯手性沸石 $\mathrm{SU}-32^{[9]}$, , 因此具有与沸石一样持久孔洞和吸附能力且合成条件 温和的手性 MOFs 受到了科学工作者的广泛关注.

本文简介近几年来手性金属有机框架材料(MOFs) 的合成及应用.

\section{1 手性 MOFs 的合成方法}

近年来, 为了开发拥有多种潜在应用价值的功能材

*E-mail: hpzeng@scut.edu.cn

Received December 26, 2011; revised March 23, 2012; published online April 27, 2012.

Project supported by the National Natural Science Foundations of China (No. 21071054), the Science and Technology Planning Project of Guangdong Province (Nos. 2008B010800030, 2009B091300045).

国家自然科学基金(No. 21071054)、广东省科技计划 (Nos. 2008B010800030，2009B091300045) 资助项目. 
料, 合理的设计和构建 MOFs 材料已经引起了人们的广 泛关注 ${ }^{[10 ~ 13]}$. 但是, 手性 MOFs 形成的最终结构很难预 测, 其原因如下：(1)中心金属离子的配位方式, 羧酸的 配位方式各异 ${ }^{[11]}$; (2)为了形成更稳定的框架结构会出现 合理的穿插结构 (溶剂的作用使穿插程度有所变 化 $)^{[10,15]}$; (3)形成晶体之间的相互作用力 (分子间的作用 力); (4)反应过程中的溶剂、温度和 $\mathrm{pH}^{[16]}$ 等的影响.

金属有机框架是由金属离子或金属簇和作为金属 中心间链接的有机配体自组装而成的具有周期性网络 结构的配位聚合物. 因此, 可以从金属的配位方式和配 体的结构两方面设计出合理的金属有机框架，而手性聚 合物只能通过手性配体或非手性配体在特殊环境中自 发转变来得到.

目前, 所合成的手性金属有机框架大致有以下三 类: (1)具有手性配体的手性金属有机框架化合物, (2)具 有次级构筑单元 (SBU)手性金属有机框架化合物, (3)具 有螺旋结构的手性金属有机框架化合物. 因此可以用下 面几种方法来设计手性 MOFs.

\section{1 通过手性配体或同分异构体合成}

合成手性框架化合物使用最广泛且最成功的方法 是直接用对映手性配体与金属桥联来得到 ${ }^{[17,18]}$, 同时同 分异构体在这方面也具有很大的潜力.

Sung 等 ${ }^{[19]}$ 用二价铜分别与 $\mathbf{L 1}$ 和 L2 (Scheme 1) 先形 成一维的棒状超分子构筑模块(SBB), 然后再堆积形成 三维的手性框架 $\mathrm{Cu}_{2}(\mathbf{1})_{2}\left(\mathrm{H}_{2} \mathrm{O}\right)_{2}(\mathrm{DMF})_{x}$ 和 $\mathrm{Cu}_{2}(\mathbf{2})_{2}(\mathrm{DMF})_{2}-$ $(\mathrm{DMF})_{x}$. 前者是薄片堆积而成的, 后者是类似夹板状的 堆积.

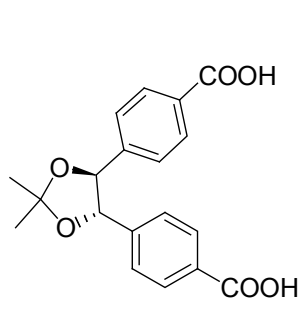

L1

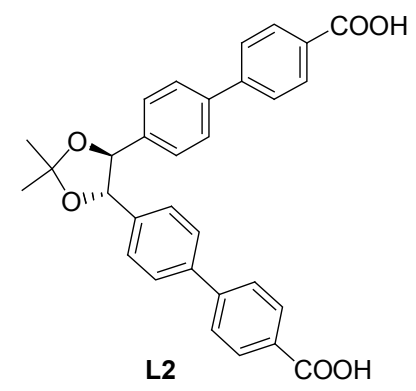

Scheme 1
Sun 等 ${ }^{[20]}$ 用硝酸锌和具有立体异构的 $\mathrm{H}_{4}$ TCPPDA (Scheme 2) 配体得到了红色块状晶体 $\left[\mathrm{Zn}_{4} \mathrm{O}\left(\mathrm{D}_{2}-\right.\right.$ $\left.\mathrm{TCPPDA})_{1.5}\right] \cdot \mathrm{DMF} \cdot \mathrm{H}_{2} \mathrm{O}$, 此结构中含有八面体形的 $\mathrm{SBU}$, 每个配体与四个 SBU 连接, 每个 SBU 与六个配 体相连形成一个三维的孔洞框架, 它是第一个具有刚玉 拓扑结构的手性 MOFs 材料.

Gedrich 等 ${ }^{[21]}$ 用连有手性基团的羧酸配体合成了 $\left[\mathrm{Zn}_{3}(\mathrm{ChirBTB}-1)_{2}(\mathrm{DEF})_{3}\left(\mathrm{H}_{2} \mathrm{O}\right)_{5}\right]$ 和 $\left[\mathrm{Zn}_{3}(\text { ChirBTB- })_{2}\right.$ $\left.(\mathrm{DEF})_{2}\left(\mathrm{H}_{2} \mathrm{O}\right)_{3}\right]$ (Scheme 3), 前者结构中的锌桨轮式的

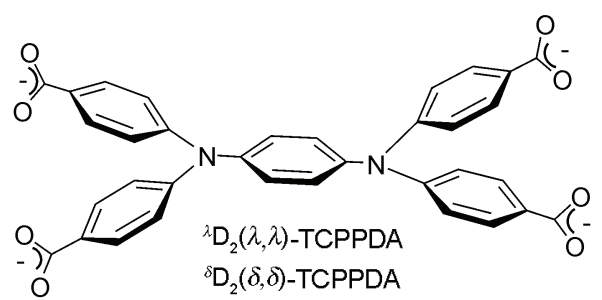

Scheme 2

次级构筑单元被四个 ChirBTB-1 配体包围, 且里面的 BTB 不是平面的而是螺旋形的, 这种结构向外延伸形 成手性的类似方嗍石的拓补结构; 后者则完全不同，三 个锌离子构成了类似线性阵列的次级构筑单元，这些 SBU 同手性链连接形成了一个三维的网状结构.

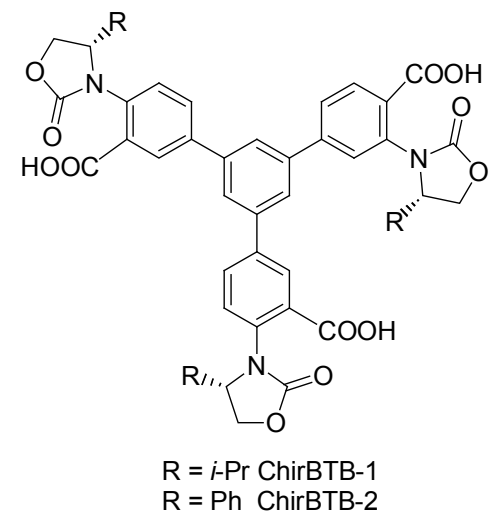

Scheme 3

$\mathrm{Wu}$ 等 ${ }^{[22]}$ 用细长的配体 $\mathbf{L 3}$ (Scheme 4)与二价金属 $(\mathrm{Zn}, \mathrm{Cd}, \mathrm{Ni})$ 合成了六个纯手性的一维和二维框架结构 (表 1), 配体中大量的苯环使几个框架结构中存在很强 的 $\pi-\pi$ 作用.

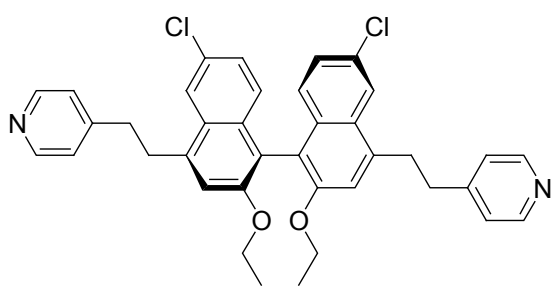

L3

\section{Scheme 4}

\section{2 利用模板分子作用合成}

模板分子在框架结构中不与金属离子桥联，但它的 结构和化学性质会影响到整个框架的结构和性质，可以 减少框架的穿插度、形成开放的孔洞结构、增加手性 等 ${ }^{[23]}$. 因此, 可以用手性模板来诱导合成手性 $\mathrm{MOFs}^{[24,25]}$.

Prior 和 Rosseinsky ${ }^{[26]}$ 首次用手性的二醇为模板合成 了手性 MOFs: $\mathrm{Ni}_{3}(\mathrm{btc})_{2}(\mathrm{py})_{6}(1,4-\mathrm{bd})_{4}(\mu-1,4-\mathrm{bd}) \cdot 2(1,4-\mathrm{bd})$ 
表 1 六个框架结构信息

Table 1 Six frame structure information

\begin{tabular}{lcc}
\hline \multicolumn{1}{c}{ 组成 } & 维数 & 颜色 \\
\hline$\left[\mathrm{Zn}(\mathrm{DMF})_{4}\right]\left[\mathrm{Zn}(\mathrm{DMF})_{2}\left(\mathrm{H}_{2} \mathrm{O}\right)\left(\mathrm{ClO}_{4}\right)\right] \cdot\left(\mathrm{ClO}_{4}\right)_{3} \cdot 2 \mathrm{DMF} \cdot \mathrm{EtOH} \cdot 2 \mathrm{H}_{2} \mathrm{O}$ & $1 \mathrm{D}$ & 无色 \\
{$\left[\mathrm{CdL}(\mathrm{DMF})_{4}\right]\left[\mathrm{CdL}(\mathrm{DMF})_{3}\left(\mathrm{ClO}_{4}\right)\right] \cdot\left(\mathrm{ClO}_{4}\right)_{3} \cdot 2 \mathrm{DMF}$} & $1 \mathrm{D}$ & 无色 \\
{$\left[\mathrm{CdL}\left(\mathrm{H}_{2} \mathrm{O}\right)\left(\mathrm{NO}_{3}\right)_{2}\right]\left[\mathrm{CdL}\left(\mathrm{H}_{2} \mathrm{O}\right)_{2}\left(\mathrm{NO}_{3}\right)_{2}\right] \cdot \mathrm{MeOH}$} & $1 \mathrm{D}$ & 无色 \\
{$\left[\mathrm{Cd}_{2} \mathrm{~L}_{4}(\mathrm{DMF})\left(\mathrm{ClO}_{4}\right)\left(\mathrm{H}_{2} \mathrm{O}\right)_{2}\right] \cdot\left(\mathrm{ClO}_{4}\right)_{3} \cdot \mathrm{DMF} \cdot 3 \mathrm{CH}_{3} \mathrm{CN} \cdot 6 \mathrm{H}_{2} \mathrm{O}$} & $2 \mathrm{D}$ & 无色 \\
{$\left[\mathrm{CdL}_{2}\left(\mathrm{NO}_{3}\right)_{2}\right] \cdot \mathrm{DMF} \cdot 2 \mathrm{EtOH} \cdot 2.5 \mathrm{H}_{2} \mathrm{O}$} & $2 \mathrm{D}$ & 无色 \\
{$\left[\mathrm{NiL}_{2} \mathrm{Cl}_{2}\right] \cdot \mathrm{DMF} \bullet 4 \mathrm{EtOH} \cdot \mathrm{H}_{2} \mathrm{O}$} & $2 \mathrm{D}$ & 亮绿色 \\
\hline
\end{tabular}

$\left(\mathrm{H}_{3} \mathrm{btc}=1,3,5\right.$-均苯三甲酸, $\mathrm{py}=$ 吡啶, $1,4-\mathrm{bd}=1,4-$-丁二 醇), 其中的模板 1,4-bd 与配体 btc 的羧酸氧形成氢键, 来确保羧酸在一个直角方向, 且每个结构单元中有四个 模板分子.

与此类似的还有无手性的 $1,3,5$-均苯三甲酸与镍离 子形成的螺旋形的 $(10,3)$ 网状结构 ${ }^{[27,28]}$, 是由手性模板 二齿醇分子束缚金属中心来连接手性框架结构的. 为了 进一步探索, Lin 和他的同事 ${ }^{[29]}$ 用手性离子液体作为媒 介合成了手性框架化合物 $(\mathrm{BMIm})_{2}\left[\mathrm{Ni}(\mathrm{Hbtc})_{2}\left(\mathrm{H}_{2} \mathrm{O}\right)_{2}\right]$ $(\mathrm{BMIm}=1$-丁基-3-甲基咪唑) .

\section{3 直接用非手性配体合成}

手性化合物的合成方法中自发产生手性结果是一 个很特殊的现象，只在 MOFs 的合成过程中发生 ${ }^{[30,31]}$, 且得到的产物多具有螺旋型的结构或次级结构单元, 其 中非手性配体中柔性的多齿有机桥联配体可能更容易 形成螺旋形的手性 $\mathrm{MOFs}^{[32]}$.

第一个光学纯的手性 MOFs 是 1999 年 Ezuhara 等 ${ }^{[33]}$ 用硝酸镉和非手性的 5-(9-蒽基)嘧啶(apd)反应得到 $\mathrm{Cd}(\mathrm{apd})\left(\mathrm{NO}_{3}\right)_{2}\left(\mathrm{H}_{2} \mathrm{O}\right)(\mathrm{EtOH})$, 框架具有镉(II)-嘧啶螺旋 链. 因为链与链之间的的氢键使相近的螺旋链呈现同样 的手性，当加入单晶晶种时就只有一种对映体.

Fang 等 ${ }^{[34]}$ 用无手性的 $\mathrm{H}_{3}$ btc $(1,3,5$-均苯三甲酸)配体 与硝酸锌反应得到 $\mathrm{Zn}_{3}(\mathrm{btc})_{2}(\mathrm{DMF})_{3}\left(\mathrm{H}_{2} \mathrm{O}\right) \cdot(\mathrm{DMF})\left(\mathrm{H}_{2} \mathrm{O}\right)$. 它是一个三维的手性开放结构框架, 含有三种不同的锌 离子, 形成两种不同的次级构筑单元：第一种锌是和来 自四个 btc 配体的五个氧原子配位, 第二种锌和来自三 个 btc 配体的六个氧原子配位, 第三种锌和来自三个 btc 配体的三个氧原子配位. 其中前两种锌一起形成一种四 面体型的次级构筑单元, 两个第三种锌通过金属键和配 体形成一种正方形的桨轮式的次级构筑单元. 这两种 SBU 通过扭曲的 btc 配体形成三维的手性框架.

Tian 等 ${ }^{[35]}$ 在无任何手性反应物、溶剂和其他辅助物 基础上用 $\mathrm{H}_{2} \mathrm{PDC}$ (吡啶-2,5-二酸)配体与醋酸铬反应得到 了 $\mathrm{Co}(\mathrm{PDC})\left(\mathrm{H}_{2} \mathrm{O}\right)_{2} \cdot \mathrm{H}_{2} \mathrm{O}$. 它是一种拥有两种类型的螺旋 链的手性二维层状结构, 配体 PDC 的每一个羧酸和两 个钴离子以顺反的配位模式形成固定的右旋 C-O-Co 链, 同时形成相反的螺旋 Co-PDC 链, 两个类型的螺旋连通
过钴离子相互连接形成二维的层结构. 从而螺旋形的手 性配合物的研究成为热点之一 ${ }^{[36 ~ 39], ~} \mathrm{Ag}(\mathrm{hat}) \mathrm{ClO}_{4}$ • $3 \mathrm{CH}_{3} \mathrm{NO}_{2}{ }^{[40]}$ (hat $=1,4,5,8,9,12$-hexaazatriphenylene) 和 $\mathrm{Zn}_{2}(\mathrm{btc})\left(\mathrm{NO}_{3}\right) \cdot \mathrm{H}_{2} \mathrm{O} \cdot 5 \mathrm{H}_{2} \mathrm{O}{ }^{[41]}\left(\mathrm{H}_{3} \mathrm{btc}=1,3,5\right.$-均苯三甲酸 $)$ 也属于这一类结构.

除以上三种主要方法外，还有一些其他的合成方 法, 如 $\left[\mathrm{Zn}_{2} \mathrm{Co}(\mathrm{tib})_{3}\left(\mathrm{H}_{2} \mathrm{O}\right)_{5}\right]\left[\mathrm{Zn}_{6}(\mathrm{tib})_{2-}(1,2,4-\mathrm{btc})_{6}\right] \cdot 12.7 \mathrm{H}_{2} \mathrm{O}$ 和 $\left[\mathrm{ZnCo}(\mathrm{tib})(1,2,4-\mathrm{btc})\left(\mathrm{H}_{2} \mathrm{O}\right)_{2}\right] \mathrm{Cl}_{3} \cdot \mathrm{H}_{2} \mathrm{O}^{[42]}[\mathrm{tib}=1,3,5-$ 三 (1-咪唑基)苯]是通过配体诱导和 $\mathrm{pH}$ 共同作用来控制合 成手性 $3 d-3 d$ 多金属 MOFS. Agt-LSbF 6 (1), Agc-LSbF 6 (2), Ag2t-L $\left.\left(\mathrm{H}_{2} \mathrm{O}\right)\right]\left(\mathrm{BF}_{4}\right)_{2}$ (3) 和 $\mathrm{Agc}_{-} \mathrm{LBF}_{4} \bullet 0.5\left(\mathrm{C}_{6} \mathrm{H}_{6}\right)$ (4) (t-L: 顺式的 L; c-L: 反式的 L $)^{[43]}$ (Scheme 5), 是手性配 体在控制温度下形成不同维数的框架结构. 还有一些手 性框架可以通过后合成修饰 ${ }^{[44]}$ 等方法来得到.

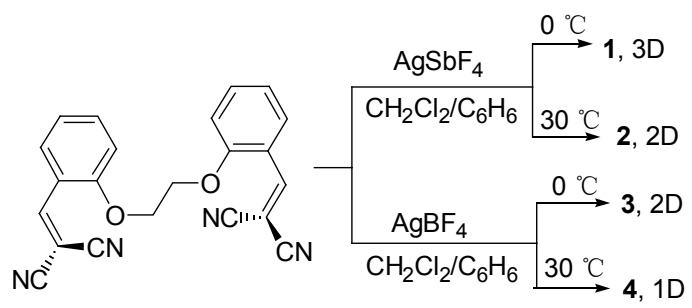

Scheme 5

\section{2 手性 MOFs 的用途}

手性金属有机框架在不对称催化、对映选择性分 离、非线性光学、手性传感、手性磁性材料、光活性纳 米级药物的传输和生物医学成像材料等方面的潜在应 用，使其成为不同学科的研究热点. 这里主要介绍不对 称催化和对映选择性分离 ${ }^{[45,46]}$.

\section{1 不对称催化}

在不对称催化反应中, 催化剂的作用是在催化中心 周围形成一个特定的空间环境，从而调整反应物分子的 空间取向，形成特定的反应通道，控制反应进程，达到 立体选择反应的目的. 因此，获得高对映选择性、高反 应活性的催化剂, 成为人们研究的重点, 而作为具有多 相不对称催化性质的手性 MOFs, 引起了化学工作者的 广泛关注。 


\subsection{1 酯基转移作用}

Seo 等 ${ }^{[7]}$ 报道了第一个具有对映选择性催化的手性 MOFs 材料 POST-1，它是通过配体 $(4 R, 5 R)-\mathbf{H L} 4$ (Scheme 6) 与硝酸锌在水和甲醇的混合溶剂中得到 $L$-POST-1, 当用其对映体时得到 D-POST-1. 此结构中 有没有配位的吡定基, 为了确保框架的中性, 有三分之 二被质子化了, 指向手性通道, 这样的吡定基使其可以 催化酯基转移反应.

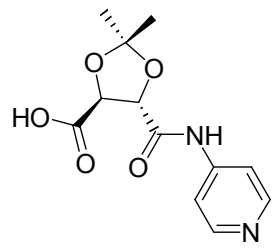

(4S,5S)-HL4

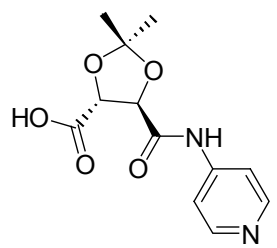

$(4 R, 5 R)-\mathrm{HL} 4$

Scheme 6

从表 2 中催化剂与吡啶比较催化酯基转移反应的结 果可以看出产物的对映选择性与纯吡啶催化相比有所 提高.

表 2 催化剂与吡啶催化酯基转移反应的结果

Table 2 The results of the catalyst and pyridine catalyzing transesterification reactions<smiles>CC(=O)Oc1ccc([N+](=O)[O-])cc1[N+](=O)[O-]</smiles>

\begin{tabular}{cccc}
\hline 催化剂 & $S / \%$ & $R / \%$ & $e e / \%$ \\
\hline$L$-POST-1 & 45.9 & 54.1 & 8.2 \\
$D$-POST-1 & 54.2 & 45.9 & 8.3 \\
吡啶 & 49.6 & 50.5 & 0.9 \\
\hline
\end{tabular}

2.1 .2 氰基硅烷化

Dang 等 ${ }^{[47]}$ 在 BCIP [N-tert-butoxy-carbonyl-2-(imidazole)-1-pyrrolidine]的手性环境中用配体 $\mathrm{H}_{4}$ MDIP [methylenediisophthalic acid] (Scheme 7)与硝酸铈反应得到<smiles>CC(C)(C)OC(=O)N1CCC[C@H]1CN1C=NCC1</smiles>

Scheme 7
具有手性孔洞的三维化合物 $\mathrm{Ce}(\mathrm{HMDIP})\left(\mathrm{H}_{2} \mathrm{O}\right)$, 去除溶 剂分子水后得到具有路易斯酸位点的框架，可用于催化 氰基烷基化反应，产物的 ee 值可达到 $98 \%$ 以上(表 3), 直接过滤后循环使用三次后催化效率仍然可以达到 $80 \%$ 以上.

表 3 MOFs 催化氰基烷基化反应结果

Table 3 The results of MOFs catalyzing the cyano alkylation reactions

\begin{tabular}{|c|c|c|}
\hline $\mathrm{O}$ & $2 \mathrm{~mol} \%$ CeMDIP & $\mathrm{OSiMe}_{3}$ \\
\hline \multirow{2}{*}{ Ar } & \multicolumn{2}{|c|}{$e e / \%$} \\
\hline & Ce-MDIP1 & Ce-MDIP? \\
\hline 苯基 & 93 & 94 \\
\hline 4-甲氧基苯基 & 91 & 97 \\
\hline 1-萘基 & 98 & 98 \\
\hline 2-䓺基 & $>98$ & $>98$ \\
\hline
\end{tabular}

\section{1 .3 羟醛缩合反应}

2009 年 Banerjee 等 ${ }^{[48]}$ 报道了手性 MOFs 催化剂 CMIL-1 和 CMIL-2, 催化此类反应 $e e$ 值可以达到 $80 \%$ 以上. 目前 Lun 等 ${ }^{[49]}$ 通过后合成修饰方法用 $\mathrm{T}$ 和硝酸锌 合成了 IRMOF-Pro-Boc $\left(\mathrm{C}_{72} \mathrm{H}_{72} \mathrm{~N}_{6} \mathrm{O}_{22} \mathrm{Zn}_{4} \cdot 7 \mathrm{H}_{2} \mathrm{O} \cdot \mathrm{DMF}\right)$, 然后加热去除 Boc 基团得到 IRMOF-Pro, ee 值达到 80\% (Scheme 8). 它可以催化差醛缩合反应(表 4), 虽然催化 效率不高, 但和前者相比还是有一定的提高, 可能是孔 洞的加大而引起的.
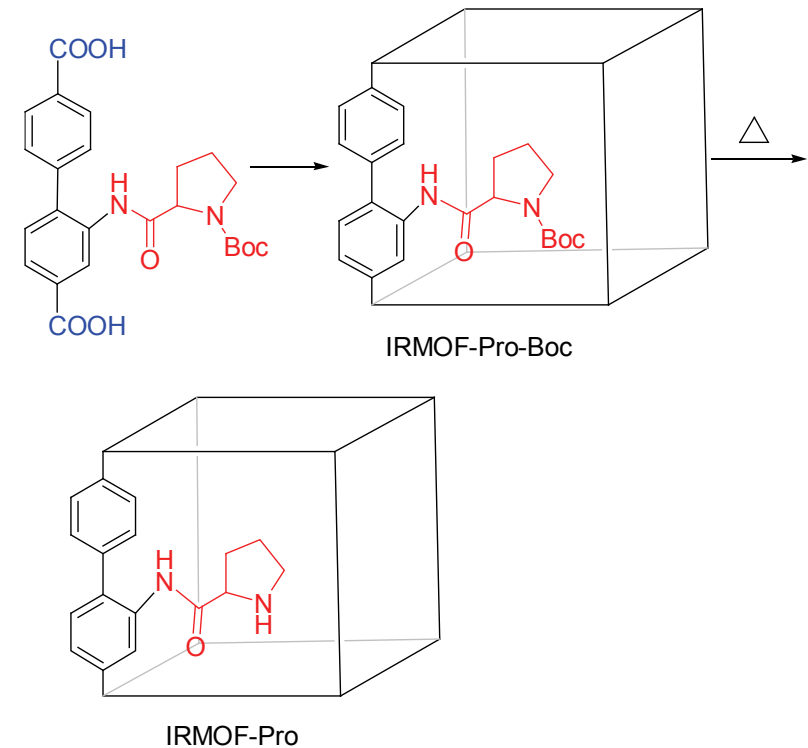

Scheme 8

\section{1 .4 二乙基锌添加到羰基碳上}

$\mathrm{Wu}$ 等 ${ }^{[50 \sim 52]}$ 从 2005 年到 2010 年先后得到了六种手 性 MOFS 用于催化二乙基锌添加到羰基碳上的反应. 它 们分别用配体 L5 L9 (Scheme 9) 与氯化铬、硝酸铜和 硝酸锌作用合成了一系列的手性多孔 MOFs 材料 
表 4 MOFs 催化羟醛缩合反应结果

Table 4 The results of MOFs catalyzing the hydroxyl aldehyde condensation reactions

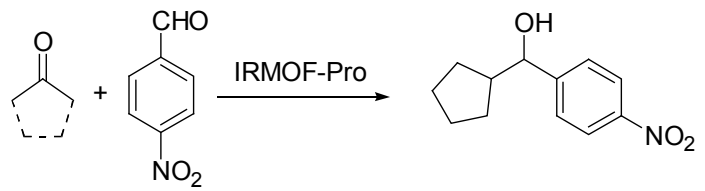

\begin{tabular}{lcc}
\hline 催化剂 & \multicolumn{2}{c}{$e e / \%$} \\
\cline { 2 - 3 } & 丙酮 & 环戊酮 \\
\hline IRMOF-Pro-Boc & 不反应 & 不反应 \\
IRMOF-Pro & 29 & $3(s y n), 14($ anti $)$ \\
\hline
\end{tabular}

(CMOF-1-6). 从表 5 中催化反应结果可以看出: 对映选 择性催化的效果与钛中心萮环立体空间大小有关.

\subsection{5 格氏反应}

Wang 等 ${ }^{[53]}$ 用手性配体(S)-3-羟基-2-(吡啶-4-甲基 胺)丙酸(HL10)与氯化铜反应生成纯手性框架 $\mathrm{Cu}_{2} \mathrm{~L}_{2} \mathrm{Cl}_{2}$, 结构中氨基功能基使其在催化格氏试剂型的反应时具 有很高的转化率和对映选择性(表 6).

\section{1 .6 狄尔斯-阿尔德反应}

Jeong 等 ${ }^{[54]}$ 用配体 $S$-L11 与硝酸铜作用得到了蓝色 块状的晶体 $\mathrm{Cu}_{2}(S-\mathbf{L 1 1})_{2}\left(\mathrm{H}_{2} \mathrm{O}\right)_{2}$, 由于它的手性环境使其 可以催化 Diels-Alder 反应, 且催化效率比单独使用配体
表 5 MOFs 的催化反应结果

Table 5 The results of MOFs catalytic reaction

\begin{tabular}{|c|c|c|c|c|}
\hline & \multirow{2}{*}{\multicolumn{2}{|c|}{ 催化剂 }} & & \\
\hline $\mathrm{Ar}$ & & & 转化率/\% & $e e / \%$ \\
\hline \multirow{7}{*}{$\mathrm{Ph}$} & \multicolumn{2}{|r|}{ CMOF-1 } & $>99$ & 83 \\
\hline & \multicolumn{2}{|r|}{ CMOF-2 } & 98 & $<3$ \\
\hline & \multicolumn{2}{|r|}{ CMOF-3 } & 98 & 70 \\
\hline & \multicolumn{2}{|r|}{ CMOF-4 } & $>99$ & 82 \\
\hline & \multicolumn{2}{|r|}{ CMOF-5 } & $>99$ & 84 \\
\hline & \multicolumn{2}{|r|}{ CMOF-6 } & 99 & 17.4 \\
\hline & \multicolumn{2}{|c|}{ 1,1'-二萗基-2,2'-二醇 } & $>99$ & 88 \\
\hline \multirow{7}{*}{ 1-Naphthyl } & \multicolumn{2}{|r|}{ CMOF-1 } & $>99$ & 93 \\
\hline & \multicolumn{2}{|r|}{ CMOF-2 } & 81 & 9 \\
\hline & \multicolumn{2}{|r|}{ CMOF-3 } & $>99$ & 86 \\
\hline & \multicolumn{2}{|r|}{ CMOF-4 } & $>99$ & 91 \\
\hline & \multicolumn{2}{|r|}{ CMOF-5 } & 60 & 91 \\
\hline & \multicolumn{2}{|r|}{ CMOF-6 } & 98 & 21.8 \\
\hline & \multicolumn{2}{|c|}{ 1,1'-二䓺基-2,2'-二醇 } & $>99$ & 94 \\
\hline
\end{tabular}

提高(表 7).

\section{1 .7 环氧化合物的胺解和醇解}

Tanaka 等 ${ }^{[55,56]}$ 用手性配体 L12 (Scheme 10) 与硝酸 铜作用得到手性的金属框架 $\left[\mathrm{Cu}_{2}(\mathbf{L 1 2})_{2}\left(\mathrm{H}_{2} \mathrm{O}\right)_{2}\right] \cdot \mathrm{MeOH}$ • $2 \mathrm{H}_{2} \mathrm{O}$ (R-3), 结构中不对称的手性环使其可以用于环氧<smiles>Oc1cc(-c2ccncc2)c2cc(Cl)ccc2c1-c1c(O)cc(-c2ccncc2)c2cc(Cl)ccc12</smiles>

L5<smiles>O=C(O)c1ccc(-c2ccc(-c3c(-c4ccc(C(=O)O)cc4)ccc4c(-c5ccc(C(=O)O)cc5)c(O)cc(-c5ccc(C(=O)O)cc5)c34)c(-c3ccccc3)c2)cc1</smiles><smiles>Cc1ccc2c(-c3c(O)cc(C(=O)O)c4cc(C(=O)O)ccc34)c(O)cc(C(=O)O)c2c1</smiles>

L6<smiles>O=C(O)C=Cc1ccc2c(-c3c(O)cc(C=CC(=O)O)c4cc(C=CC(=O)O)ccc34)c(O)cc(C=CC(=O)O)c2c1</smiles>

L7<smiles>O=C(O)c1ccc(/C=C/c2ccc3c(-c4c(O)cc(/C=C/c5ccc(C(=O)O)cc5)c5cc(/C=C/c6ccc(C(=O)O)cc6)ccc45)c(O)cc(/C=C/c4ccc(C(=O)O)cc4)c3c2)cc1</smiles>

Scheme 9 
表 6 MOFs 催化格氏试剂型反应结果

Table 6 The results of MOFs catalytic reaction of Grignard reagents<smiles>[R]C(=[O+])/C=C/c1ccc([R])cc1</smiles><smiles>[R]c1ccc(/C=C/C([R])(O)C2CCCCC2)cc1</smiles>

\begin{tabular}{cccc}
\hline $\mathrm{R}^{1}$ & $\mathrm{R}^{2}$ & 转化率/\% & $e e / \%$ \\
\hline $\mathrm{Me}$ & $\mathrm{Me}$ & 93 & $>99$ \\
$\mathrm{Cl}$ & $\mathrm{Me}$ & 94 & 97 \\
$\mathrm{H}$ & $\mathrm{H}$ & 88 & 55 \\
$\mathrm{H}$ & $\mathrm{Me}$ & 97 & 65 \\
\hline
\end{tabular}

表 7 MOFs 催化 Diels-Alder 反应的结果

Table 7 The results of MOFs catalyzing Diels-Alder reaction<smiles>C=C(CCOC)OC</smiles><smiles>O=C1C=COC(c2ccccc2)C1</smiles><smiles>Cc1cc(C(=O)O)cc(O)c1-c1c(C)cc(C(=O)O)cc1O</smiles>

(S)-L11

\begin{tabular}{lcc}
\hline \multicolumn{1}{c}{ 催化剂 } & 转化率/\% & $e e / \%$ \\
\hline $\mathrm{Cu}_{2}(S-\mathbf{L 1 1})_{2}\left(\mathrm{H}_{2} \mathrm{O}\right)_{2}$ & 80 & 55 \\
$S$-L11 & 72 & 51 \\
$R$-L11 & 58 & 18 \\
\hline
\end{tabular}

化合物的胺解(表 8)和醇解(表 9), 胺解时无溶剂且烷基 链越长, 效率越高.<smiles>CCCC(=O)c1cccc2c(-c3c(O)ccc4c(C(=O)O)cccc34)c(O)ccc12</smiles>

Scheme 10

用手性配体, Ingleson 等 ${ }^{[57]}$ 等合成了一系列不同天 冬氨酸的 MOFs, 其中具有柱层结构的纯手性 $\mathrm{Cu}(L-$ asp)(bpe $)_{0.5}\left(\mathrm{H}_{2} \mathrm{O}\right)_{0.5}(\mathrm{MeOH})_{0.5} \quad[L-\mathrm{asp}=$ 天冬氨酸; bpe $=$ 1,2-二(4-吡啶基)乙烯]在乙醚中用无水氯化氢后合成得
表 8 MOFs 的胺解反应结果

Table 8 The results of MOFs aminolysis reaction

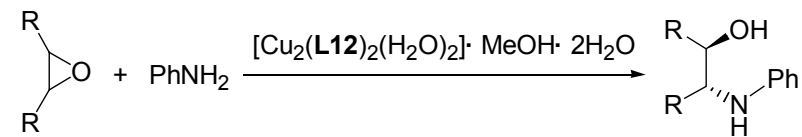

a: $\mathrm{R}=\left(\mathrm{CH}_{2}\right)_{4}, \mathbf{b}: \mathrm{R}=\left(\mathrm{CH}_{2}\right)_{3}$

\begin{tabular}{cccc}
\hline 环氧化物 & 溶剂 & 转化率 $/ \%$ & $e e / \%$ \\
\hline $\mathbf{a}$ & 甲苯 & 54 & 45 \\
$\mathbf{b}$ & 甲苯 & 15 & 43 \\
$\mathbf{a}$ & - & 51 & 51 \\
$\mathbf{b}$ & - & 30 & 50 \\
\hline
\end{tabular}

表 9 MOFs 的醇解反应结果

Table 9 The results of MOFs alcoholysis reaction

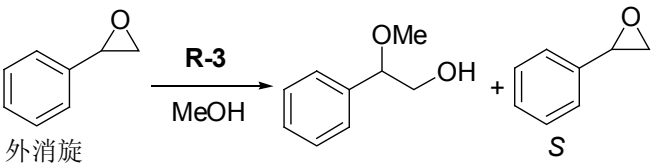

\begin{tabular}{cccccc}
\hline \multirow{2}{*}{ 环氧化物 } & \multicolumn{2}{c}{ 产物 } & & \multicolumn{2}{c}{ 未反应的反应物 } \\
\cline { 2 - 3 } \cline { 5 - 6 } & 转化率 $/ \%$ & $e e / \%($ 构型 $)$ & & 转化率 $/ \%$ & $e e / \%($ 构型 $)$ \\
\hline 外消旋 & 20 & $62(S)$ & & 64 & $19(S)$ \\
$S$ & 48 & $>99(S)$ & & 50 & $>99(R)$ \\
$R$ & 5 & $>99(R)$ & & 92 & $>99(S)$ \\
\hline
\end{tabular}

到框架 $\mathrm{Cu}(L$-asp $) \mathrm{bpe}_{0.5}(\mathrm{HCl})\left(\mathrm{H}_{2} \mathrm{O}\right)$, 它可以催化环氧化 合物的醇解(表 10 ), 虽然产率比 $\mathrm{H}_{2} \mathrm{SO}_{4}$ 催化时低很多(它 的孔洞太小了, 不利于反应物的扩散), 但 ee 值有所提 高.

表 10 MOFs 的醇解反应结果

Table 10 The results of MOFs alcoholysis reaction

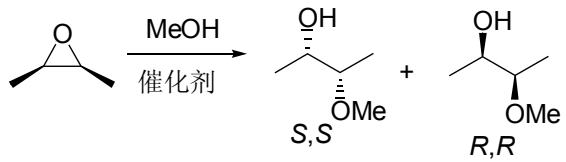

\begin{tabular}{cccc}
\hline 催化剂 & 温度 $/{ }^{\circ} \mathrm{C}$ & 转化率 $/ \%$ & $e e / \%$ \\
\hline$L$ 型 & 25 & 59 & 10 \\
$D$ 型 & 25 & 65 & -6 \\
$L$ 型 & 0 & 30 & 17 \\
$D$ 型 & 0 & 32 & -13 \\
硫酸 & 25 & 100 & 2 \\
\hline
\end{tabular}

2.1.8 酮的氢化反应

在 2003 年 $\mathrm{Hu}$ 等 ${ }^{[58]}$ 磷酸类配体 L13, L14 (Scheme 11 ) 分别与 $\left(\mathrm{RuPhCl}_{2}\right)_{2},(R, R)$-二苯基乙二胺和 $\mathrm{Zr}(\mathrm{OBu})_{4}$ 反 应得到两个手性结构 $\mathrm{Zr}\left[\mathrm{Ru}(\mathrm{L} 13)(\mathrm{DPEN}) \mathrm{Cl}_{2}\right] \cdot 4 \mathrm{H}_{2} \mathrm{O}$ (Zr-Ru-L13)和 $\mathrm{Zr}\left[\mathrm{Ru}(\mathbf{L 1 4})(\mathrm{DPEN}) \mathrm{Cl}_{2}\right] \cdot 4 \mathrm{H}_{2} \mathrm{O}(\mathrm{Zr}-\mathrm{Ru}-\mathbf{L 1 4})$ [DPEN: 二苯基乙二胺], 对芳香酮的催化加氢反应具有 很高的对映选择性, ee 值最高达到 $99 \%$ 以上(表 11). 
<smiles>O=P(O)(O)c1cc(-c2ccccc2)c(-c2c(-c3ccccc3)cc([PH](=O)(=O)O)c3ccccc23)c2ccccc12</smiles>

L13<smiles>O=Pc1ccc2c(-c3ccccc3)c(-c3ccccc3)ccc2c1</smiles>

L14

\section{Scheme 11}

表 11 MOFs 催化芳香酮的加氢反应结果

Table 11 The results of MOFs catalyzing the aromatic ketone of hydrogenation reactions

\begin{tabular}{|c|c|c|}
\hline \multirow{2}{*}{$\mathrm{Ar}$} & \multicolumn{2}{|c|}{$e e / \%$} \\
\hline & Zr-Ru-L13 & Zr-Ru-L14 \\
\hline $\mathrm{Ph}$ & 96.3 & 79.0 \\
\hline 2-Naphthyl & 97.1 & 82.1 \\
\hline 4'- $-\mathrm{BuC}_{6} \mathrm{H}_{4}$ & 99.2 & 91.5 \\
\hline 4'- $\mathrm{MeOC}_{6} \mathrm{H}_{4}$ & 96.0 & 79.9 \\
\hline $4^{\prime}-\mathrm{ClC}_{6} \mathrm{H}_{4}$ & 94.9 & 59.3 \\
\hline 4'- $-\mathrm{MeC}_{6} \mathrm{H}_{4}$ & 97.0 & 79.5 \\
\hline 2-Naphthyl & 99.2 & 95.8 \\
\hline
\end{tabular}

\subsection{9 烯烃的环氧化}

Abraham ${ }^{[59]}$ 和 Cho 等 ${ }^{[60]}$ 用同样的配体 L15 分别与 L16 和 bpdc (图 12)在硝酸锌的作用下得到了手性的 $\mathrm{Zn}_{2}(\mathrm{bpdc})_{2} \mathbf{L 1 5}$ 和 MnSO-MOF, 它们都可以催化烯烃的 环氧化(表 12), 并可以直接过滤后循环使用, 且多次后 催化效率仍然很好.

表 12 烯烃的环氧化反应结果

Table 12 The results of epoxidation of olefins<smiles>CCCCOc1ccccc1O</smiles>

\begin{tabular}{lcc}
\hline \multicolumn{1}{c}{ 催化剂 } & 转化率/\% & ee/\% \\
\hline $\mathbf{L 1 5}$ & - & 90 \\
$\mathrm{Zn}_{2}(\mathrm{bpdc})_{2} \mathbf{L 1 5}$ & 71 & 88 \\
$\mathrm{MnSO}_{\mathrm{MOF}}$ & 40 & 80 \\
\hline
\end{tabular}

以上不对称烯烃的环氧化反应中起催化作用的是 框架中的活性金属位点和孔洞. 但是框架中的次级构筑 单元易水解不稳定, 使其催化性能不高, 通过加入 2-(叔 丁基磺酰基)亚碘酰苯作为氧化剂可以提高反应活性.

Song 等 ${ }^{[61,62]}$ 用前面同一类型的配体 $\mathbf{L 1 7} \sim \mathbf{L 2 0}$ 合成 了一系列的手性 MOFS $\left[\mathrm{Zn}_{4}\left(\mu_{4}-\mathrm{O}\right)(\mathbf{L 1 7})_{3}\right] \cdot 20 \mathrm{DMF} \cdot 2 \mathrm{H}_{2} \mathrm{O}$ (CMOF-1), $\left[\mathrm{Zn}_{4}\left(\mu_{4}-\mathrm{O}\right)(\mathbf{L 1 8})_{3}\right] \cdot 42 \mathrm{DMF}(\mathrm{CMOF}-2),\left[\mathrm{Zn}_{4^{-}}\right.$ $\left.\left(\mu_{4}-\mathrm{O}\right)(\mathbf{L 1 8})_{3}\right] \cdot 37 \mathrm{DEF} \cdot 23 \mathrm{EtOH} \cdot 4 \mathrm{H}_{2} \mathrm{O}(\mathrm{CMOF}-3),\left[\mathrm{Zn}_{4}\left(\mu_{4^{-}}\right.\right.$ $\left.\mathrm{O})(\mathbf{L 1 9})_{3}\right] \cdot 38 \mathrm{DMF} \cdot 11 \mathrm{EtOH} \quad(\mathrm{CMOF}-4), \quad\left[\mathrm{Zn}_{4}\left(\mu_{4}-\mathrm{O}\right)-\right.$ $\left.(\mathbf{L 2 0})_{3}\right] \cdot 40 \mathrm{DBF} \cdot 6 \mathrm{EtOH} \cdot \mathrm{H}_{2} \mathrm{O}(\mathrm{CMOF}-5)$, 同样可以催化 环氧化反应(表 13).

手性 MOFs 作为催化剂具有分离简单、可循化使用、 可利用孔洞大小选择性限制、提供晶体结构(更准确的了 解孔洞的结构、特性以及催化活性位点的分布)等多相催 化剂的优点, 它与绿色化学追求的实现高效、高选择性 (包括化学选择、区域选择、立体选择和对映选择)的化 学反应、极少的副产物和实现零排放以达到原子经济性

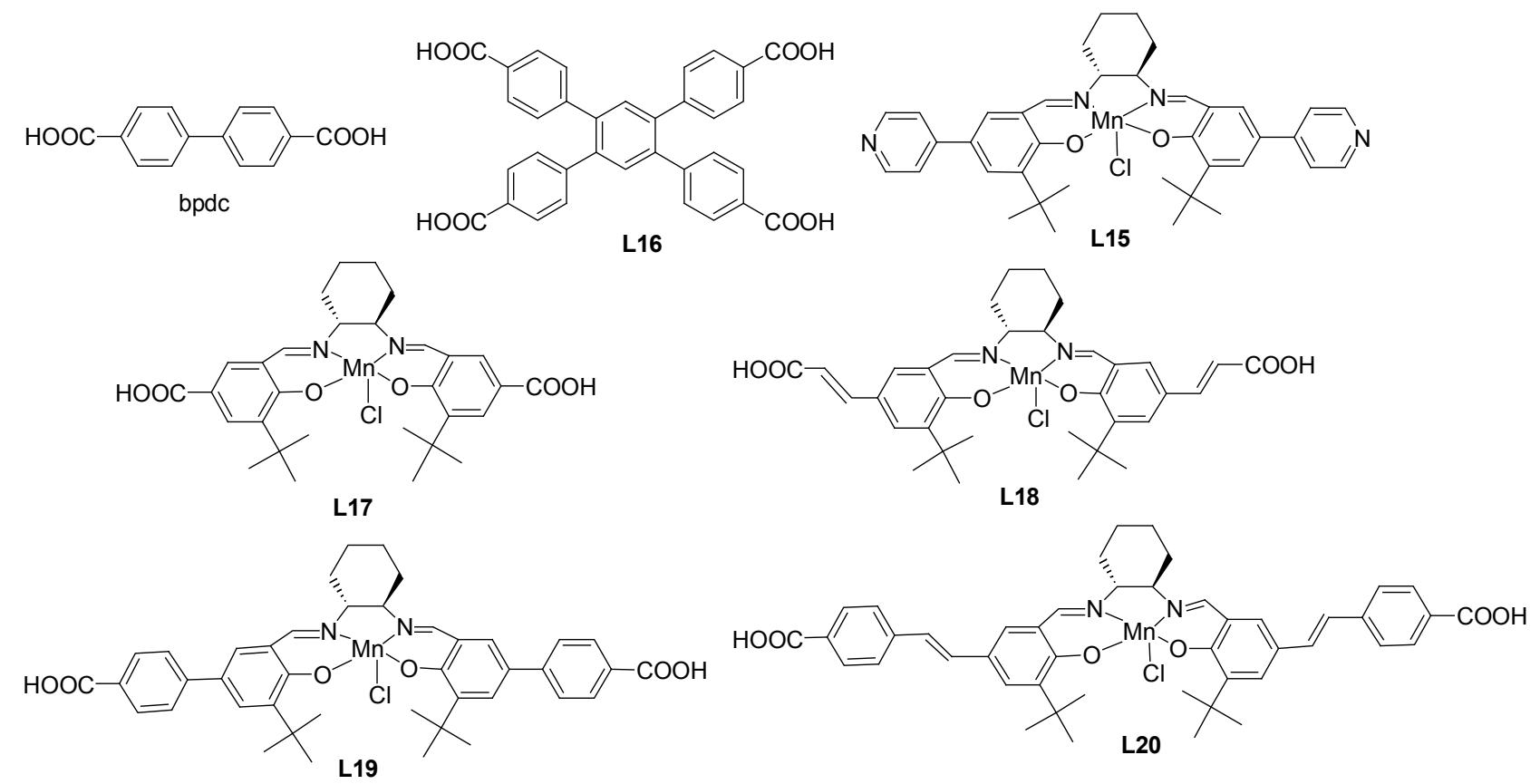

Scheme 12 
表 13 几种不同的 MOFs 催化环氧化反应的结果

Table 13 The results of several different MOFs catalyzing epoxidation reactions<smiles>[R]C=C[R]</smiles>

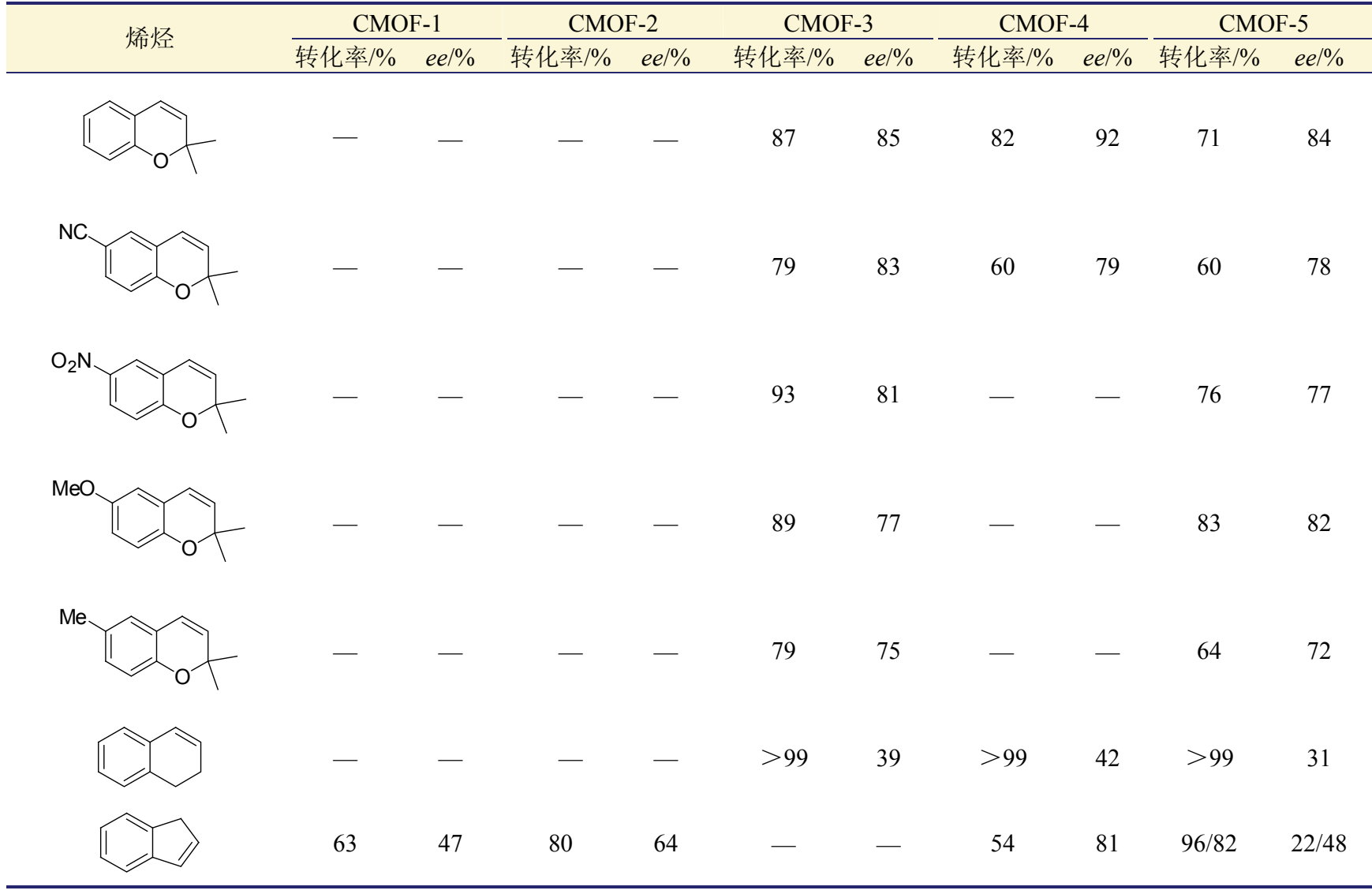

反应的目标相适应，另外运用不对称催化合成低成本、 高药效的手性药物一直是科学工作者关注的，而还有很 多反应类型没有被探索，因此，手性 MOFs 作为不对称 催化反应的催化剂仍具有很大的发展潜力.

\section{2 对映选择性分离}

自然界的很多物质以对映体存在，而有用的只是其 中的某一个对映异构体, 这时就需要将两种物质分离, 而其物理性质非常相似, 不可能直接用纯物理的方法分 离, 因此需要找一个合适的介质. 手性 MOFs 因为它的 手性孔洞或其中的含有的手性官能团而引起了人们的 关注，成为外消旋体拆分的候选者.

Vaidhyanathan 等 ${ }^{[63]}$ 用天冬氨酸 ( $L$-asp), 4,4'-联吡啶 (bpy)和硝酸镍反应得到三维的柱结构 $\mathrm{Ni}_{2}(L \text {-asp })_{2}$ bpy, 可以用于分离手性的二醇(表 14).

另外，表 15 中的几种手性结构也可以用来分离一 些对映体.

近年来手性 MOFs 已经做成色谱柱用于外消旋体拆
表 $14 \mathrm{MOFs}$ 用于分离二醇的结果

Table 14 The results of MOFs to separate diol

\begin{tabular}{ccc}
\hline 二醇 & $e e / \%$ & 标准 $e e / \%$ \\
\hline 1,2-丙二醇 & 5.35 & 0.16 \\
1,2-丁二醇 & 5.07 & 0.88 \\
2,3-丁二醇 & 1.50 & 0.50 \\
1,3-丁二醇 & 17.93 & 0.62 \\
1,2-戊二醇 & 13.90 & 0.34 \\
2,4-戊二醇 & 24.5 & 0 \\
2-甲基-2,4-戊二醇 & 53.77 & 0.46 \\
1,2-己二醇 & 5.00 & 0.09 \\
2,5-己二醇 & 3.40 & 0 \\
\hline
\end{tabular}

分, 2007 年首次用锌金属框架作为高效液相色谱的固定 相在外消旋的混合物中分离手性的烷基芳基亚砜 ${ }^{[66]}$, 目 前也已有可用于气相色谱分离的固定相的手性 MOFs. Xie 等 ${ }^{[67]}$ 报道了用醋酸铜和 $\mathrm{H}_{2} \mathrm{sala}[N$-(2-邻差茎苄基)- $L$-丙 氨酸]合成的单螺旋配位聚合物 $\left\{[\mathrm{Cu}(\mathrm{sala})]_{2}\left(\mathrm{H}_{2} \mathrm{O}\right)\right\}_{n}$ 加热 去除水以后，交叉连接产生了一个手性的开放孔洞，用 它作为气相色谱分离的固定相对烷烃、醇类、同分异 
表 15 不同的 MOFs 分离不同的物质的结果 ${ }^{a}$

Table 15 The result of different MOFs to separate the different substances

\begin{tabular}{|c|c|c|c|c|c|c|}
\hline 框架结构 & 配体 & 金属 & 结构 & 分离物 & $e e / \%$ & 参考文献 \\
\hline 二维层状多孔结构 & $(4 R, 5 R)-\mathbf{H L 4}$ & 硝酸锌 & $L$-POST-1 & {$\left[\mathrm{Ru}\left(2,2^{\prime} \text {-bipy }\right)_{3}\right] \mathrm{Cl}_{3}$} & 66.0 & {$[7]$} \\
\hline 手性开放孔洞 & QA & 氢氧化镉 & $\mathrm{Cd}(\mathrm{QA})_{2}$ & $\begin{array}{l}\text { 2-丁醇 } \\
\text { 2-甲基-1-丁醇 }\end{array}$ & $\begin{array}{c}98.2 \\
8.4\end{array}$ & {$[64]$} \\
\hline 手性螺旋 & Btc, pic, pd & 硝酸镍 & $\mathrm{Ni}_{3}(\mathrm{btc})_{2}(\mathrm{pic})_{6}(\mathrm{pd})_{3}$ & 联萘酚 & 8.3 & {$[65]$} \\
\hline
\end{tabular}

${ }^{a}$ QA: 6-甲氧基-(8S,9R)-金鸡纳-9-醇-3-羧酸; btc: 均苯三酸; pic: 3-甲基吡啶;pd: 1,2-丙二醇.

构体有很好的识别能力, 尤其是手性化合物. 其中, 在 手性分离中起作用的是配体的手性碳.

含有金属簇或第二个金属离子构成 MOFs 不仅能够 形成可调节的孔洞、弯曲形状和手性孔洞环境, 而且可 以有不同的固定金属位点(开放金属位点、催化活性金属 位点和光活性位点), 是一种多功能的框架结构. Das 等 ${ }^{[68]}$ 用金属配体 $\mathrm{Cu}(\mathrm{SalPyCy})$ 和 $\mathrm{H}_{2} \mathrm{bdc}$ (对苯甲二酸)或 $\mathrm{H}_{2} \mathrm{cdc}$ (对环已烷二甲酸)与锌离子一起合成了三维手性 的 $\left[\mathrm{Zn}_{3}(\mathrm{bdc})_{3}\{\mathrm{Cu}(\mathrm{SalPyCy})\}(\mathrm{G})_{x}\right]$ 和 $\left[\mathrm{Zn}_{3}(\mathrm{cdc})_{3}\{\mathrm{Cu}(\mathrm{SalPy}-\right.$ $\mathrm{Cy})\}(\mathrm{G})_{x}$ ] (Scheme 13), 已经被用于手性识别和分离 1苯基乙醇, 可以吸收 $S$-1-苯基乙醇, 从而达到对映体分 离的目的, 因为后者的孔洞尺寸小于前者, 从而分离能 力提高了很多.

在实验室和工业生产中很多时候我们得到的是外 消旋体, 但当两个产品的作用明显不同的时候, 就需要 我们能够得到光学纯的产品(尤其是医药和农药方面),
而作为对映体分离的候选者 MOFs, 仍然需要我们不但 去开发具有良好分离能力的新结构.

\section{3 展望}

手性 MOFs 由于其构筑特殊，性能独特，引起人们

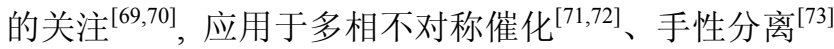
等. 虽然催化效率、分离效果等仍未达到我们期望的结 果, 但是对于催化剂的回收使用是其他方法不可企及 的. 手性 MOFs 具有较高的药物承载能力、生物降解性 等多方面的功能表现出色，但作为手性药物的储存和传 递的载体, 要求具有很高的生物兼容性、无毒性和纳米 级的尺寸(在血液里循环应达到的)等, 还需要我们去探 索、研究. 除此之外, 作为显影剂的菼光材料、核磁共 振的磁性材料 ${ }^{[74]}$ 、非线性光学材料和传感材料等方面仍 需要进一步发展.

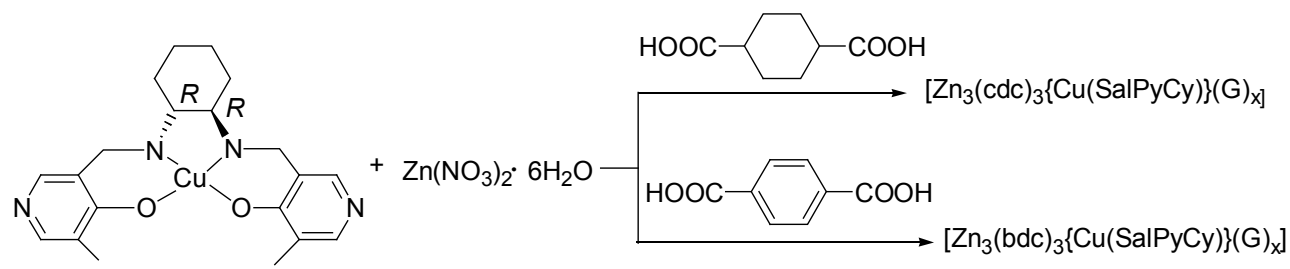

Scheme 13

\section{References}

[1] Hoskins, B. F.; Robson, R. J. Am. Chem. Soc.1990, 112, 1546.

[2] Robson, R.; Abrahams, B. F.; Batten, S. R.; Gable, R. W.; Hoskins, B. F.; Liu, J. P. ACS Symp. Ser. 1992, 499, 256.

[3] Ji, M. W.; Lu, P.; Liu, J.; Wang, M.; Liu, F. J.; Xia, G. M. Shandong Chem. Ind. 2011, 40, 42 (in Chinese).

(纪穆为, 卢萍, 刘静, 王敏, 刘凤静, 夏光明, 山东化工, 2011, 40, 42.)

[4] Liu, H.; Zhao, Y. G.; Zhang, Z. L.; Nijem, N.; Chabal, Y. J.; Zeng, H. P.; Li, J. Adv. Funct. Mater. 2011, 21, 4754.

[5] Zhao, Y. G.; Wu, H. H.; Emge, T. J.; Gong, Q. H.; Nijem, N.; Chabal, Y. J.; Kong, L. Z.; Langreth, D. C.; Liu, H.; Zeng, H. P.; Li, J. Chem. Eur. J. 2011, 17, 5101.

[6] Li, K. H.; Olson, D. H.; Seidel, J.; Emge, T. J.; Gong, H. W.; Zeng, H. P.; Li, J. J. Am. Chem. Soc. 2009, 131, 10368

[7] Seo, J. S.; Whang, D.; Lee, H.; Jun, S. I.; Oh, J.; Jeon, Y. J.; Kim, K. Nature 2000, 404, 982.

[8] Castillo, J. M.; Vlugt, J. H.; Dubbeldam, D.; Hamad, S.; Calero, S. J. Phys. Chem. C 2010, 114, 22207.
[9] Zhang, L.; Jiang, J. J. Membr. Sci. 2011, 367, 63.

[10] Eddaoudi, M.; Moler, D. B.; Li, H. L.; Hen, B. L.; Reineke, T. M.; O'Keeffe, M.; Yaghi, O. M. Acc. Chem. Res. 2001, 34, 319.

[11] Ockwig, N. W.; Delgado-Friedrichs, O.; O'Keeffe, M.; Yaghi, O. M. Acc. Chem. Res. 2005, 38, 176.

[12] Natarajan, S.; Mahata, P. Chem. Soc. Rev. 2009, 38, 2304.

[13] Alexandrov, E. V.; Blatov, V. A.; Kochetkov, A. V.; Proserpio, D. M. Cryst. Eng. Commun. 2011, 13, 3947.

[14] Wei, W. Y.; Fang, J.; Kong, H. N.; Han, J. Y.; Chang, H. Y. Prog. Chem. 2005, 17, 1110 (in Chinese).

(魏文英，方键，孔海宁，韩金玉，常贺英，化学进展，2005，17， 1110.)

[15] Kesanli, B.; Cui, Y.; Smith, M. R.; Bittner, E. W.; Bockrath, B. C.; Lin, W. Angew. Chem. 2005, 117, 74.

[16] Su, Z.; Fan, J.; Okamura, T.; Sun, W. Y.; Ueyama, N. Cryst. Growth Des. 2010, 10, 3515.

[17] Cui, Y.; Evans, O. R.; Ngo, H. L.; White, P. S.; Lin, W. Angew. Chem., Int. Ed. 2002, 41.

[18] Kristina, G.; Irena, S.; Igor, A. B.; Uwe, M.; Oliver, T.; Stefan, K. Inorg. Chem. 2010, 49, 4440. 
[19] Sung, M. S.; Dohyun, M.; Kyung, S. J.; Jaheon, K.; Praveen, K. T.; Nakcheol, J. Chem. Commun. 2011, 47, 9402.

[20] Sun, D. F.; Collins, D. J.; Ke, Y. X.; Zou, J. L.; Zhou, H. C. Chem. Eur. J. 2006, 12, 3768.

[21] Gedrich, K.; Heitbaum, M.; Notzon, A.; Senkovska, I.; Fröhlich, R.; Getzschmann, J.; Mueller, U.; Glorius, F.; Kaskel, S. Chem. Eur. J. 2011, 17, 2099.

[22] Wu, C. D.; Zhang, L.; Lin, W. B. Inorg. Chem. 2006, 45, 7278.

[23] Daisuke, T.; Susumu, K. Chem. Mater. 2008, $20,922$.

[24] Zhang, J.; Liu, R.; Feng, P.; Bu, X. Angew. Chem., Int. Ed. 2007, 46,8388 .

[25] An, H.; Wang, E.; Xiao, D.; Li, Y.; Su, Z.; Xu, L. Angew. Chem., Int. Ed. 2006, 45, 904.

[26] Prior, T. J.; Rosseinsky, M. J. Inorg. Chem. 2003, 42, 1564.

[27] Kepert, C. J.; Prior, T. J.; Rosseinsky, M. J. J. Am. Chem. Soc. 2000, 122, 5158 .

[28] Bradshaw, D.; Prior, T. J.; Cussen, E. J.; Claridge, J. B.; Rosseinsky, M. J. J. Am. Chem. Soc. 2004, 126, 6106.

[29] Lin, Z.; Slawin, A. M. Z.; Morris, R. E. J. Am. Chem. Soc. 2007, $129,4880$.

[30] Jacques, J.; Collet, A.; Wilen, S. H. Enantiomers, Racemates and Resolutions, Krieger Publishing Company, Malabar, FL, 1994.

[31] Pérez-García, L.; Amabilino, D. B. Chem. Soc. Rev. 2002, 31, 342.

[32] Wang, X. W.; Han, L.; Cai, T. J.; Zheng, Y. Q.; Chen, J. Z.; Deng, Q. Cryst. Growth Des. 2007, 7, 1027.

[33] Ezuhara, T.; Endo, K.; Aoyama, Y. J. Am. Chem. Soc. 1999, 121, 3279.

[34] Fang, Q. R.; Zhu, G. S.; Xue, M.; Sun, J. Y.; Sun, F. X.; Qiu, S. L. Inorg. Chem. Commun. 2006, 45, 3582.

[35] Tian, G.; Zhu, G. S.; Yang, X. Y.; Fang, Q. R.; Xue, M.; Sun, J. Y.; Wei, Y.; Qiu, S. L. Chem. Commun. 2005, 1396.

[36] Biradha, K.; Seward, C.; Zaworotko, M. J. Angew. Chem., Int. Ed 1999, 48, 492.

[37] Batten, S. R.; Hoskins, B. F.; Robson, R. Angew. Chem., Int. Ed. Engl. 1997, 36, 636.

[38] Gao, Q.; Jiang, F. L.; Wu, M. Y.; Huang, Y. G.; Wei, W.; Hong, M. C. Cryst. Growth Des.. 2010, 10, 184.

[39] Beatriz, G. H.; Henning, A. H.; Jana, K. V.; Joaquin, S.; Christoph, J. Chem. Commun. 2010, 46, 8270.

[40] Abrahams, B. E.; Jackson, P. A.; Robson, R. Angew. Chem., Int. Ed. 1998, 37, 2656.

[41] Yaghi, O. M.; Davis, C. E.; Li, G. M.; Li, H. L. J. Am. Chem. Soc. 1997, 119, 2861 .

[42] Su, Z.; Fan, J.; Okamura, T. A.; Sun, W. Y.; Ueyama, N. Cryst. Growth Des. 2010, 10, 3515.

[43] Dong, Y. B.; Jiang, Y. Y.; Li, J.; Ma, J. P.; Liu, F. L.; Tang, B.; Huang, R. Q.; Batten, S. R. J. Am. Chem. Soc. 2007, 129, 4520.

[44] Banerjee, M.; Das, S.; Yoon, H.; Choi, H. J.; Hyun, M. H.; Park, S. M.; Seo, G.; Kim, K. J. Am. Chem. Soc. 2009, 131, 7524.

[45] Liu, Y.; Xuan, W. M.; Cui, Y. Adv. Mater. 2010, 22, 4112.

[46] Nickerl, G.; Henschel, A.; Grünker, R.; Gedrich, K.; Kaskel, S. Chem. Ing. Tech. 2011, 83, 90.
[47] Dang, D. B.; Wu, P. Y.; He, C.; Xie, Z.; Duan, C. Y. J. Am. Chem. Soc. 2010, 132, 14321.

[48] Banerjee, M.; Das, S.; Yoon, M.; Choi, H. J.; Hyun, M. H.; Park, S. M.; Seo, G.; Kim, K. J. Am. Chem. Soc. 2009, 131, 7524.

[49] Lun, D. J.; Waterhouse, G. I. N.; Telfer, S. G. J. Am. Chem. Soc. 2011, 133, 5806 .

[50] Wu, C. D.; Hu, A.; Zhang, L.; Lin, W. J. Am. Chem. Soc. 2005, 127,8940

[51] Ma, L.; Falkowski, J. M.; Abney, C.; Lin, W. Nat. Chem. 2010, 2, 838.

[52] Ma, L.; Wu, C. D.; Wanderley, M. M.; Lin, W. Angew. Chem., Int. Ed. 2010, 49, 8244.

[53] Wang, M.; Xie, M. H.; Wu, C. D.; Wang, Y. G. Chem. Commun. 2009, 17, 2396.

[54] Jeong, K. S.; Go, Y. B.; Shin, S. M.; Lee, S. J.; Kim, J.; Yaghi, O. M.; Jeong, N. Chem. Sci. 2011, 2, 877.

[55] Tanaka, K.; Oda, S.; Shiro, M. Chem. Commun. 2008, 820.

[56] Tanaka, K; Otani, K. New J. Chem. 2010, 34, 2389.

[57] Ingleson, M. J.; Barrio, J. P.; Bacsa, J.; Dickinson, C.; Park, H.; Rosseinsky, M. J. Chem. Commun. 2008, 11, 1287.

[58] Hu, A.; Ngo, H. L.; Lin, W. B. J. Am. Chem. Soc. 2003, 125, 11490 .

[59] Abraham, M. S.; Omar, K. F.; Debashis, A.; Amy, A. S.; Joseph, T. H.; SonBinh, T. N. Inorg. Chem. 2011, 50, 3174.

[60] Cho, S. H.; Ma, B. Q.; Nguyen, S. B. T.; Hupp, J. T.; Albrecht-Schmit, T. E. Chem. Commun. 2006, 2563.

[61] Song, F. J.; Wang, C.; Lin, W. B. Chem. Commun. 2011, 47, 8256.

[62] Song, F. J.; Wang, C.; Falkowski, J. M.; Ma, L. Q.; Lin, W. B. J. Am. Chem. Soc. 2010, 132, 15390.

[63] Vaidhyanathan, R.; Bradshaw, D.; Rebilly, J. N; Barrio, J. P.; Gould, J. A.; Berry, N. G.; Rosseinsky, M. J. Angew. Chem., Int Ed. 2006, 45, 6495 .

[64] Xiong, R. G.; You, X.; Abrahams, B. F.; Xue, Z.; Che, C. Angew. Chem., Int. Ed. 2001, 40, 4422.

[65] Bradshaw, D.; Prior, T. J.; Cussen, E. J.; Claridge, J. B.; Rosseinsky, M. J. J. Am. Chem. Soc. 2004, 126, 6106.

[66] Nuzhdin, A. L.; Dybtsev, D. N.; Bryliakov, K. P.; Talsi, E. P.; Fedin, V. P. J. Am. Chem. Soc. 2007, 129, 12958.

[67] Xie, S. M.; Zhang, Z. J.; Wang, Z. Y.; Yuan, L. M. J. Am. Chem. Soc. 2011, 133, 11892

[68] Das, M. C.; Xiang, S. C.; Zhang, Z. J.; Chen, B. L. Angew. Chem., Int. Ed. 2011, 50, 10510.

[69] Li, H. Y.; Jiang, L.; Xiang, H.; Makal, T. A.; Zhou, H. C.; Lu, T. B. Inorg. Chem. 2011, 50, 3177.

[70] Gao, Q.;Wang, X. Q.; Jacobson, A. J. Inorg. Chem. 2011, 50, 9073.

[71] Yoon, M.; Srirambalaji, R.; Kim, K. Chem. Rev. 2011.

[72] Wang, C.; Zheng, M.; Lin, W. B. J. Phys. Chem. Lett. 2011, 2, 1701.

[73] Tan, Y. X.; He, Y. P.; Zhang, J. Inorg. Chem. 2011, 50, 11527.

[74] Nagaraja, C. M.; Haldar, R.; Maji,T. K.; Rao, C. N. R. Cryst. Growth Des. 2012, 12, 975. 